\title{
Internal Carotid Artery S-Shaped Curve as a Marker of Fibromuscular Dysplasia in Dissection-Related Acute Ischemic Stroke
}

\author{
Diogo C. Haussen ${ }^{a}$ Ashutosh Jadhav ${ }^{b}$ Leticia C. Rebello ${ }^{a}$ \\ Samir Belagaje $^{a}$ Aaron Anderson $^{a}$ Tudor Jovin $^{b} \quad$ Amin Aghaebrahim $^{b}$ \\ Deepak Gulati $^{b}$ Bryan Wells ${ }^{c}$ Michael Frankel ${ }^{a}$ Raul G. Nogueira ${ }^{a}$ \\ ${ }^{a}$ Emory University School of Medicine/Marcus Stroke and Neuroscience Center - \\ Grady Memorial Hospital, Atlanta, Ga., ' University of Pittsburgh Medical Center, \\ Pittsburgh, Pa., and 'Emory University School of Medicine, Atlanta, Ga., USA
}

\section{Key Words}

Dissection · Carotid stenting $\cdot$ Carotid occlusive disease $\cdot$ Carotid artery $\cdot$ Stroke

\begin{abstract}
Background and Purpose: Craniocervical fibromuscular dysplasia (FMD) is associated with dissections and with S-shaped curves in the internal carotid artery (ICA). We evaluated the occurrence of S-curves in patients presenting with acute strokes due to ICA steno-occlusive dissections. Methods: This was a retrospective review of the interventional databases of two academic tertiary-care institutions. The presence of ICA S-shaped curves, C-shaped curves, 360-degree loops, as well as classic FMD and atherosclerotic changes at the ICA bulb and curve/loop was determined. Cases of carotid dissections were compared with a control group (consecutive non-tandem anterior circulation strokes). Results: Twenty-four patients with carotid dissections were compared to 92 controls. Baseline characteristics and procedural variables were similar, with the exception of younger age, less frequent history of hypertension, diabetes, atrial fibrillation and stent retriever use in patients with dissections. The rates of mTICI2b-3 reperfusion, parenchymal hematoma, good outcome and mortality were similar amongst groups. The frequency of S-curves (any side without superimposed atherosclerosis) was $29 \%$ in the dissection group versus $7 \%$ in controls $(p<0.01$ ). S-curves were typically mirror images within the dissection group ( $85 \%$ had bilateral occurrence). The frequency of Cshaped and 360-degree curves was similar between groups. FMD changes within the craniocervical arteries were statistically more common in dissection patients. Ten patients (41\%) of the dissection group had S-curves or classic FMD changes. Multivariate analysis indicated that S-curves were independently associated with the presence of dissections. Conclusion: Sshaped ICA curves are predictably bilateral, highly associated with carotid dissections in patients with moderate to severe strokes, and may suggest an underlying presence of FMD.
\end{abstract}




\section{Introduction}

Fibromuscular dysplasia (FMD) is more common than previously recognized [1]. The percentage of internal carotid artery (ICA) involvement in FMD has been reported as 74\% in a recent US registry [1]. Craniocervical FMD has been clearly associated with dissections, predisposed by the underlying vessel wall alternating segments of thickened fibromuscular hyperplasia and thinning of media with superimposed intimal hyperplasia [2].

However, FMDs have a relatively broad histopathological and radiological spectrum [1-3]. A recent study reported that the presence of an S-shaped curve in the ICA is 17 times more frequent in patients with a diagnosis of FMD as compared to age-matched controls [4]. Considering that ICA redundancy has been associated with dissections [5, 6], we evaluated the occurrence and characteristics of S-shaped curves as a marker of FMD in patients presenting with moderate to severe acute ischemic strokes (AIS) due to ICA steno-occlusive dissections.

\section{Methods}

\section{Patient Selection}

Dissection Cases

This was a retrospective review of the interventional databases of two academic tertiary care institutions for all cases with angiographically confirmed spontaneous or traumatic carotid dissections leading to AIS between September 2010 and March 2015 (Emory University/Grady Memorial Hospital) and between 2012 and 2014 (University of Pittsburgh Medical Center). Inclusion criteria encompassed the presence of high-grade ( $\geq 70 \%$ stenosis by NASCET criteria) or occlusive carotid dissections. Patients were excluded if the dissection was iatrogenic or intradural. Aortic arch and innominate dissections extending into the carotids, as well as dissections involving the vertebrobasilar system were also excluded. Baseline noncontrast CT was used to grade the Alberta Stroke Program Early CT Score (ASPECTS) [7]. The reperfusion rate was graded as per modified Treatment In Cerebral Ischemia (mTICI) [8]. Hemorrhagic complications were categorized as per European Cooperative Stroke Study (ECASS) criteria [9]. This study was approved by the local Institutional Review Board.

Controls

The control group was constituted by consecutive cases of anterior circulation large-vessel AIS, with either ICA terminus, middle cerebral artery M1 or M2 occlusion, absence of steno-occlusive extracranial ICA disease, absence of dissections (spontaneous, traumatic or iatrogenic) who underwent head-and-neck CT angiography (CTA) at some point during hospitalization at Emory University/Grady Memorial Hospital. A total of 109 patients were screened from December 2012 to September 2014; 9 were excluded due to poorquality CTA and 8 due to the presence of a contralateral ICA steno-occlusive lesion, leaving 92 patients for analysis.

\section{Imaging Criteria}

The carotid curves were defined after biplane angiography evaluation and/or CTA coronal/sagittal/ axial maximal intensity projection evaluation (3D reconstructions not uniformly available and therefore not utilized). A S-shaped curve was characterized by an elongation of the mid-to-distal ICA causing two markedly tortuous turns in the shape of the letter ' $S$ ' [4]. The proximal angle had to be less than 90 degrees, and with two relatively parallel segments within the curve (fig. 1). A C-shaped curve was defined as per two tortuous turns in the shape of the letter ' $C$ ' (first angle $\geq 90$ degrees and second angle <90 degrees) (fig. 2). A 360-degree loop was defined as per a curve with a relatively constant angle $>90$ degrees (fig. 2). Isolated kinks (fig. 2) leading to luminal narrowing were not recorded as abnormal. Areas of tortuosity with minor angulations that did not fit the aforementioned criteria were not recorded.

All CTAs were analyzed in coronal/sagittal/axial planes for the presence of atherosclerotic changes at the level of the ICA bulb and at the level of the curve (if present). Atherosclerosis was defined as present or absent according to the verification of calcifications in plaques. Catheter angiograms and CTAs were eval- 

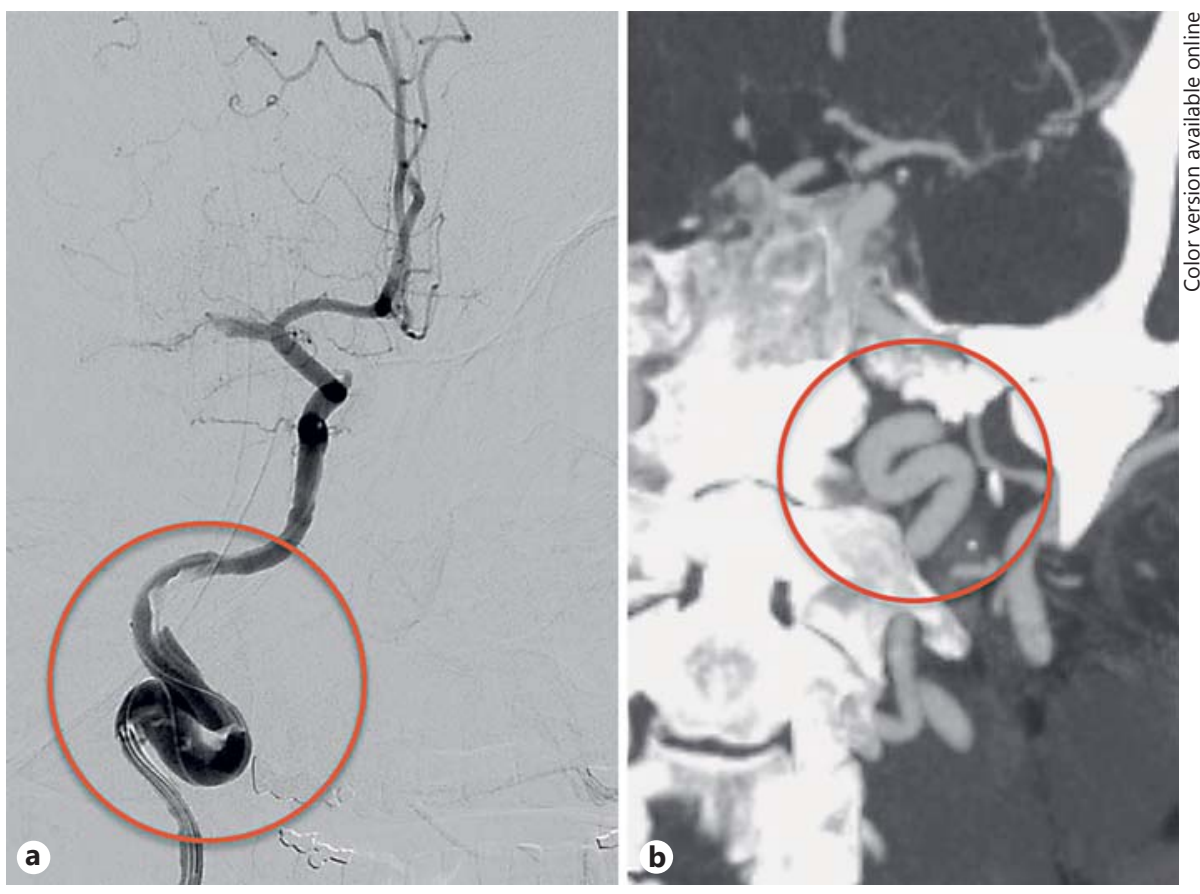

Fig. 1. Classification of ICA morphological types: the S-shaped curve. a Frontal internal carotid angiogram revealing an S-shaped curve. b Coronal CTA demonstrating an S-shaped curve.

uated for the presence of curves, classic FMD changes (string of beads) [1], and for cerebral aneurysms. Since the dissected arteries were manipulated during the endovascular treatment, resulting in spasm, recanalization with residual intraluminal thrombus or persistent occlusion, the index carotid was never utilized for assessment of FMD changes.

\section{Statistical Analysis}

Continuous variables are reported as mean \pm SD or median [IQR]. Categorical variables are reported as proportions. Between groups, comparisons for continuous/ordinal variables were made with the Student $t$ test, Mann-Whitney U test or ANOVA, as appropriate. Categorical variables were compared by $\chi^{2}$ or Fisher's exact test as appropriate. Significance was set at $p<0.05$. The primary outcome was predictors of the occurrence of S-shaped curves on any side without superimposed atherosclerosis (since superimposed atherosclerotic changes could indicate a mixed pathophysiological process) [10]. The secondary outcome was predictors of dissections. Multivariate logistic regression analysis was performed for variables at the 0.1 level of significance on univariate analysis, using a variable selection method. Statistical analyses were performed using IBM $^{\circledR}$ SPSS $^{\circledR}$ Statistics $21\left(\right.$ IBM $^{\circledR}$, Armonk, N.Y., USA). p values $<0.05$ were considered statistically significant.

\section{Results}

A total of 24 patients with carotid dissections were identified and compared to 92 controls. The baseline characteristics were similar, with the exception of expected younger age and less prevalence of hypertension, diabetes and atrial fibrillation in the dissection group (table 1). Procedural variables were also similar, with the exception of a large percentage of the use of stent retrievers in the control group. The rates of mTICI $2 \mathrm{~b}-3$ reperfusion, parenchymal hematomas, good outcomes and mortality were similar amongst the two groups (table 1). 

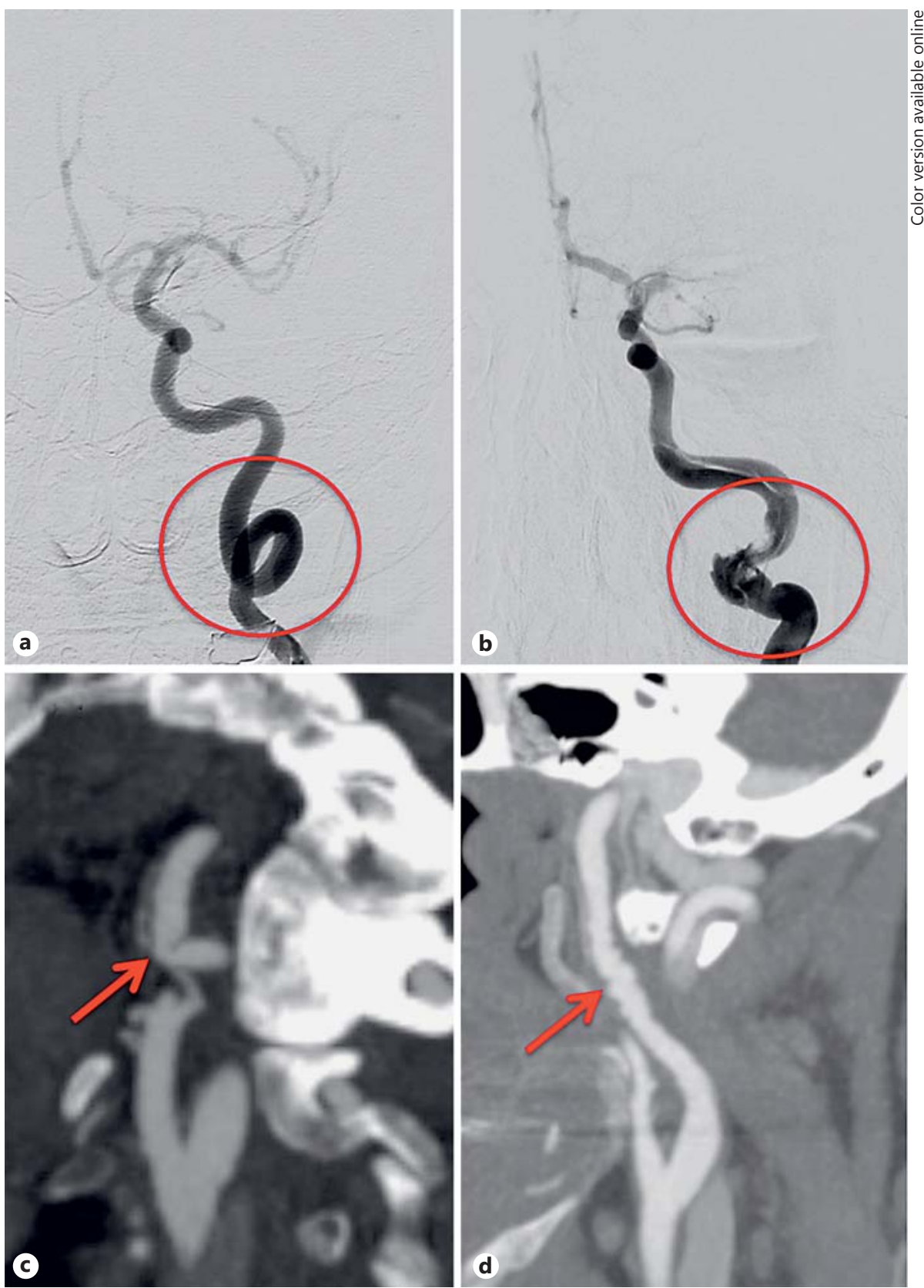

Fig. 2. Classification of other ICA morphological types. a Frontal angiogram with carotid 360-degree loop. b Frontal angiogram indicating a C-shaped curve. c Sagittal CTA revealing internal carotid kinking. d Sagittal CTA with classic FMD changes.

The inter-rater correlation coefficient for the presence of FMD was assessed $(n=30$; rho $=0.70 ; p<0.01)$, for the presence of different ICA curves ( $n=84 ;$ rho $=0.52 ; p<0.01)$, as well as, specifically, for S-shaped curves $(n=84 ;$ rho $=0.50 ; p<0.01)$. The occurrence of the different types of curves within the dissection and control groups is displayed in table 2. After excluding cases with superimposed ICA atherosclerotic changes, the frequency of S-shaped curves in any side was $29 \%$ in the dissection group versus $7 \%$ in the control group $(\mathrm{p}<0.01)$. The S-shaped ICA curves without atherosclerosis were typically mirror images within the 
Table 1. Baseline characteristics, radiologic and procedural varialbles

Haussen et al.: Internal Carotid Artery S-Shaped Curve as a Marker of Fibromuscula Dysplasia in Dissection-Related Acute Ischemic Stroke

\begin{tabular}{lccr}
\hline & $\begin{array}{c}\text { Dissections } \\
(\mathrm{n}=24)\end{array}$ & $\begin{array}{c}\text { Controls } \\
(\mathrm{n}=92)\end{array}$ & $\mathrm{p}$ value \\
\hline Age, years & $51.6 \pm 11.9$ & $63.1 \pm 15.4$ & $<0.01$ \\
Male gender & $18(75)$ & $52(56)$ & 0.10 \\
Hypertension & $9(37)$ & $65(70)$ & $<0.01$ \\
Dyslipidemia & $5(21)$ & $39(42)$ & 0.05 \\
Diabetes & $2(8)$ & $37(40)$ & $<0.01$ \\
Smoking & $0(0)$ & $10(11)$ & 0.08 \\
Atrial fibrillation & $2(8)$ & $37(40)$ & $<0.01$ \\
Intravenous tPA & $11(52)$ & $46(50)$ & 0.82 \\
ASPECTS & $7.7 \pm 1.7$ & $7.9 \pm 1.4$ & 0.62 \\
NIHSS & $17.3 \pm 5.7$ & $17.2 \pm 5.8$ & 0.76 \\
Procedure length, h & $1.8 \pm 1.0$ & $1.3 \pm 0.9$ & 0.06 \\
Last normal - puncture, h & $5.1 \pm 2.2$ & $6.2 \pm 4.3$ & 0.10 \\
\hline Intracranial occlusion & & & \\
$\quad$ ICA-T & $4(16)$ & $17(18)$ & 1.00 \\
$\quad$ MCA M1 & $11(46)$ & $52(56)$ & 0.36 \\
$\quad$ MCA M2 & $5(21)$ & $24(26)$ & 0.79 \\
Stent retriever & $14(58)$ & $83(90)$ & $<0.01$ \\
Reperfusion mTICI2b-3 & $21(87)$ & $76(82)$ & 0.12 \\
Reperfusion mTICI3 & $7(29)$ & $47(51)$ & 0.15 \\
\hline Hemorrhage & & & \\
$\quad$ PH-1 & $0(0)$ & $6(6)$ & 0.20 \\
$\quad$ PH-2 & $0(0)$ & $4(4)$ & 0.29 \\
mRS $\leq 2$ at 90 days & $16(66)$ & $47(51)$ & 0.81 \\
Mortality at 90 days & $10(11)$ & 0.52 \\
\hline
\end{tabular}

Values are means \pm SD or $\mathrm{n}(\%)$. tPA = Alteplase; NIHSS = National Institutes of Health Stroke Scale; Last normal - puncture = time interval from last known normal to groin puncture; ICA-T = ICA terminus; MCA = middle cerebral artery; $\mathrm{PH}=$ parenchymal hemorrhage; $\mathrm{mRS}=$ modified Rankin Scale.

dissection group (85\% of the patients with S-shaped curves had bilateral occurrence). The frequency of C-shaped and 360-degree curves was statistically similar between groups, and none had bilateral occurrence within the dissection group. Classic FMD changes within the craniocervical arteries were statistically more common in the patients with dissections. Ten patients (41\%) of the dissection group had either classic FMD changes or an S-Shaped curve. Twenty patients with dissections had follow-up vascular imaging. Out of the 6 patients with S-curves (1 stented), 3 (50\%) reoccluded (including the stented patient), while of the 14 patients without S-curves (6 stented), 3 (21\%) reoccluded (3 stented). No cerebral aneurysms were observed.

Multivariate analysis for the occurrence of S-Shaped curves without superimposed atherosclerosis in patients with cervical steno-occlusive dissections demonstrated no statistical association with National Institutes of Health Stroke Scale (NIHSS) (OR 0.90; 95\% CI $0.80-1.00 ; \mathrm{p}=0.05$ ) or procedural times (OR 1.01;95\% CI1.00-1.02; $\mathrm{p}=0.07$ ) after adjustment. Age was not found to impact the occurrence of S-shaped curves. S-shaped curves were independently associated with the presence of dissections (OR 4.83; 95\% CI 1.39-16.79; $\mathrm{p}=0.01$ ). 
Table 2. ICA morphological features

\section{DOI: $10.1159 / 000447978$}

C) 2016 S. Karger AG, Basel www.karger.com/ine

Haussen et al.: Internal Carotid Artery S-Shaped Curve as a Marker of Fibromuscular Dysplasia in Dissection-Related Acute Ischemic Stroke

\begin{tabular}{|c|c|c|c|}
\hline & $\begin{array}{l}\text { Dissections } \\
(n=24)\end{array}$ & $\begin{array}{l}\text { Controls } \\
(n=92)\end{array}$ & $\begin{array}{l}\mathrm{p} \\
\text { value }\end{array}$ \\
\hline \multicolumn{4}{|l|}{ S-shaped curve } \\
\hline Ipsilateral & $6(25)$ & $8(8)$ & 0.04 \\
\hline Contralateral & $7(35)^{*}$ & $10(11)$ & 0.01 \\
\hline Any side & $7(29)$ & $13(14)$ & 0.04 \\
\hline \multicolumn{4}{|l|}{ Any side - without } \\
\hline atherosclerosis & 7 (29) & $7(7)$ & $<0.01$ \\
\hline Bilateral & $6(25)$ & $5(5)$ & $<0.01$ \\
\hline \multicolumn{4}{|l|}{ Bilateral - without } \\
\hline atherosclerosis & $5(21)$ & $2(2)$ & $<0.01$ \\
\hline \multicolumn{4}{|l|}{ C-shaped curve } \\
\hline Ipsilateral & $3(12)$ & $3(3)$ & 0.06 \\
\hline Contralateral & $1(5)^{*}$ & $6(6)$ & 0.87 \\
\hline Any side & $3(12)$ & $7(7)$ & 0.36 \\
\hline \multicolumn{4}{|l|}{ 360-degree curve } \\
\hline Ipsilateral & $3(12)$ & $2(2)$ & 0.02 \\
\hline Contralateral & $0(0)^{*}$ & $2(2)$ & 0.52 \\
\hline Any side & $3(12)$ & $4(4)$ & 0.10 \\
\hline \multicolumn{4}{|l|}{ Atherosclerosis } \\
\hline \multicolumn{4}{|l|}{ Bulb } \\
\hline Ipsilateral & $0(0)$ & $26(28)$ & $<0.01$ \\
\hline Contralateral & $1(5)^{*}$ & $30(32)$ & 0.02 \\
\hline \multicolumn{4}{|l|}{ Curve } \\
\hline Ipsilateral & $0(0)$ & $4(4)$ & 0.29 \\
\hline contralateral & $1(5)^{*}$ & $1(1)$ & 0.20 \\
\hline Classic FMD changes & $5(21)^{*}$ & $2(2)$ & $<0.01$ \\
\hline Cerebral aneurysm & $0(0)^{*}$ & $0(0)$ & - \\
\hline
\end{tabular}

Values are $\mathrm{n}(\%) .{ }^{*}$ Four contralateral ICAs could not be evaluated in the dissection group due to the lack of CTA or catheter angiography images.

\section{Discussion}

Our main study finding was that S-shaped curves without superimposed atherosclerosis are typically bilateral and independently associated with carotid dissections in patients with large-vessel acute stroke undergoing thrombectomy. Close to half of the patients with dissections in our series had either classic FMD changes or S-shaped curves.

Curves in the ICA are common in postmortem or angiographic statistics [10]. The challenge resides in the fact that definitions are variable and overlap [4, 10-14]. Therefore, the interpretation of frequencies and its implications are challenging. Many different etiologies have been suggested for carotid elongations including congenital variations, aging, atherosclerosis, or collagen structural disease; however, concrete data remains limited [10]. Nonetheless, growing evidence has linked the presence of ICA anomalies (looping, curving, and kinking) with connective tissue diseases. A report evaluating the histopathology of the resected samples of 10 individuals revascularized due to ICA elongation, demonstrated that all specimens had reduction of elastic fibers and muscular cells with a compensatory increase in well-differentiated connective tissue [15]. Another study evaluated 92 patients with symptomatic carotid elongation and revealed that all examined arterial specimens demonstrated 
non-atherosclerotic and non-inflammatory histological findings primarily involving the media and intima [2]. Over half of these patients unequivocally demonstrated either typical or atypical histological patterns of FMD [2]. Recently, a cohort of 116 patients included in the US Registry for FMD revealed the S-shaped curve (as defined in the present study) as a novel and distinct morphological pattern of the mid-distal ICA in FMD [4]. Our findings seem to indicate that a large proportion of patients with large-vessel AIS related to carotid dissection may have FMD as underlying etiology.

The presence of carotid elongation has been associated with dissections [6, 16]. Our findings corroborate the notion that elongation (specifically S-curve) is independently associated with dissection. It is unclear if this observation may indicate a predisposition for thrombotic-thromboembolic phenomena due to flow impairment/shear wall stress, if the curves simply denote the underlying presence of abnormal wall histopathology (which would predispose the wall to dissections), or both. S-shaped curves were observed to be typically bilateral. This is a helpful finding since during thrombectomy in patients with ICA occlusions, a challenging course of the non-opacified carotid can be possibly predicted by analysis of the contralateral side, facilitating intra-procedural planning/navigation.

Our study has limitations inherent to its retrospective design. As discussed, definitions for curves are notoriously confusing. Furthermore, the moderate degree of correlation between two independent readers in our study corroborates the difficulty for accurate classification when more stringent definitions are used. The lack of healthy individuals and of patients with asymptomatic dissections, as well as the exclusion of patients with atherosclerotic steno-occlusive ICA disease from the control group could have introduced bias.

\section{Conclusion}

S-shaped ICA curves are predictably bilateral, highly associated with carotid dissections in patients undergoing thrombectomy for AIS, and may suggest the underlying presence of FMD.

\section{Disclosure Statement}

D.C.H., A.J., L.C.R., SB, Aaron Anderson, Amin Aghaebrahim, D.G., B.W. and M.F. report no conflicts of interest related to this study. T.J: consultant with Praxair and Silk Road Medical. R.G.N.: Stryker Neurovascular (Trevo-2 Trial PI, DAWN Trial PI), Covidien (SWIFT and SWIFT-PRIME Steering Committee, STAR Trial Core Lab), and Penumbra (3-D Separator Trial Executive Committee). There was no funding for this work.

\section{References}

1 Olin JW, Froehlich J, Gu X, Bacharach JM, Eagle K, Gray BH, Jaff MR, Kim ES, Mace P, Matsumoto AH, McBane RD, Kline-Rogers E, White CJ, Gornik HL: The United States Registry for Fibromuscular Dysplasia: results in the first 447 patients. Circulation 2012;125:3182-3190.

2 Ballotta E, Thiene G, Baracchini C, Ermani M, Militello C, Da Giau G, Barbon B, Angelini A: Surgical vs medical treatment for isolated internal carotid artery elongation with coiling or kinking in symptomatic patients: a prospective randomized clinical study. J Vasc Surg 2005;42:838-846; discussion 846.

3 Joux J, Chausson N, Jeannin S, Saint-Vil M, Mejdoubi M, Hennequin JL, Deschamps L, Smadja D, Olindo S: Carotid-bulb atypical fibromuscular dysplasia in young Afro-Caribbean patients with stroke. Stroke 2014;45: 3711-3713.

4 Sethi SS, Lau JF, Godbold J, Gustavson S, Olin JW: The S curve: a novel morphological finding in the internal carotid artery in patients with fibromuscular dysplasia. Vasc Med 2014;19:356-362.

5 Luken MG 3rd, Ascherl GF Jr, Correll JW, Hilal SK: Spontaneous dissecting aneurysms of the extracranial internal carotid artery. Clin Neurosurg 1979;26:353-375. 
Haussen et al.: Internal Carotid Artery S-Shaped Curve as a Marker of Fibromuscular Dysplasia in Dissection-Related Acute Ischemic Stroke

6 Barbour PJ, Castaldo JE, Rae-Grant AD, Gee W, Reed JF 3rd, Jenny D, Longennecker J: Internal carotid artery redundancy is significantly associated with dissection. Stroke 1994;25:1201-1206.

7 Barber PA, Demchuk AM, Zhang J, Buchan AM: Validity and reliability of a quantitative computed tomography score in predicting outcome of hyperacute stroke before thrombolytic therapy. ASPECTS Study Group. Alberta Stroke Programme Early CT Score. Lancet 2000;355:1670-1674.

8 Zaidat 00, Yoo AJ, Khatri P, et al; Cerebral Angiographic Revascularization Grading (CARG) Collaborators, STIR Revascularization working group, STIR Thrombolysis in Cerebral Infarction (TICI) Task Force: Recommendations on angiographic revascularization grading standards for acute ischemic stroke: a consensus statement. Stroke 2013;44:2650-2663.

9 Berger C, Fiorelli M, Steiner T, Schabitz WR, Bozzao L, Bluhmki E, Hacke W, von Kummer R: Hemorrhagic transformation of ischemic brain tissue: asymptomatic or symptomatic? Stroke 2001;32:1330-1335.

10 Paulsen F, Tillmann B, Christofides C, Richter W, Koebke J: Curving and looping of the internal carotid artery in relation to the pharynx: frequency, embryology and clinical implications. J Anat 2000;197:373-381.

11 Weibel J, Fields WS: Tortuosity, coiling, and kinking of the internal carotid artery. II. Relationship of morphological variation to cerebrovascular insufficiency. Neurology 1965;15:462-468.

12 Metz H, Murray-Leslie RM, Bannister RG, Bull JW, Marshall J: Kinking of the internal carotid artery. Lancet 1961;1:424-426.

13 Vannix RS, Joergenson EJ, Carter R: Kinking of the internal carotid artery. Clinical significance and surgical management. Am J Surg 1977;134:82-89.

14 Vollmar J, Nadjafi AS, Stalker CG: Surgical treatment of kinked internal carotid arteries. Br J Surg 1976;63: 847-850.

15 La Barbera G, La Marca G, Martino A, Lo Verde R, Valentino F, Lipari D, Peri G, Cappello F, Valentino B: Kinking, coiling, and tortuosity of extracranial internal carotid artery: is it the effect of a metaplasia? Surg Radiol Anat 2006;28:573-580.

16 Saba L, Argiolas GM, Sumer S, Siotto P, Raz E, Sanfilippo R, Montisci R, Piga M, Wintermark M: Association between internal carotid artery dissection and arterial tortuosity. Neuroradiology 2015;57:149-153. 